\title{
Is Seasonal Variation Another Risk Factor for Postpartum Depression?
}

\author{
Veena Panthangi, MD, Patricia West, PhD, Ruth T. Savoy-Moore, PhD, \\ Manjeet Geeta, MD, and Eileen Reickert, MD
}

Introduction: Postpartum depression (PPD) occurs in the first 6 months after delivery in $10 \%$ to $20 \%$ of mothers. Despite the availability of screening tools, there is a general consensus that PPD is underdiagnosed. A number of risk factors contributing to PPD have been investigated, but role of seasonal variability in PPD is unclear. Our purpose was to assess whether seasonal variation is another risk factor for PPD.

Methods: This cross-sectional pilot study was conducted at 2 family medicine clinics and an obstetrics/gynecology clinic over 24 months. During their postpartum visit, mothers who gave consent were asked to fill out a survey requesting demographic data, followed by the Edinburgh Postpartum Depression Scale (EPDS). The EPDS is a well-validated tool shown to be highly effective in detecting postnatal depression. A score $>12$ on the EPDS indicated a likely risk of PPD.

Results: Of the 556 patients approached, 530 completed the EPDS. Mean ( \pm SE) patient age was $24.9 \pm 0.2$ years; $71 \%$ were African Americans; $74 \%$ were single mothers; and $39 \%$ had at least some college education. On the EPDS, $17.8 \%$ scored $\geq 13$. Of the depressed patients, $18.1 \%$ had babies born during the winter, $19.2 \%$ had babies born during the spring, $13.4 \%$ during the summer, and $21.5 \%$ during autumn $\left(\chi^{2} ; P=.342\right)$. Mothers with very good or excellent support at home had lower EPDS scores $(12.6 \%)$ than mothers with just adequate support (44.0\%) or very little or no support $(30.8 \%$; $P<.0005)$. A greater proportion of women with a history of depression $(42.9 \%$ vs $12.9 \%)$ or who were currently taking antidepressives $(58.3 \%$ vs $15.9 \%)$ were in the depressed group $(P<.0005)$. Logistic regression analysis with the above variables, excluding education and income (excess missing data), on the $\mathbf{4 5 2}$ women with complete datasets found 4 significant predictors of an EPDS score $>12$. Predictors were history of depression (odds ratio [OR], 4.003; 95\% CI, 2.016-7.949); parity (OR, 1.431; 95\% CI, 0.204-1.701); social support (OR, 3.904; 95\% CI, 2.08-7.325); and currently taking medication for depression (OR, 3.613; 95\% CI, 1.207-10.817).

Conclusion: The slight seasonal variation in PPD in our pilot study was not statistically significant. Our study was underpowered to detect the projected differences in seasons. Additional patients are needed to diversify the participants and provide an adequate sample to test the projected seasonal differences. The high ORs found for greater parity, weak social support, history of depression, and currently taking antidepressants suggest that new mothers with these characteristics should be questioned about symptoms of PPD. (J Am Board Fam Med 2009;22:492-497.)

Postpartum depression (PPD) is a serious, identifiable, and treatable condition. Symptoms start within 4 weeks of childbirth and may include sleep

This article was externally peer reviewed.

Submitted 2 April 2008; revised 18 June 2009; accepted 26 June 2009.

From the Departments of Family Medicine (VP, PW, MG, ER) and Medical Education (RTS-M), St. John Hospital and Medical Center, Detroit, MI

Funding: St. John Hospital and Medical Center Graduate Medical Education Research Committee.

Conflict of interest: none declared.

Corresponding author: Veena Panthangi, MD, Family Medical Department, St John Hospital and Medical Center, Family Medicine, 24911 Little Mack Avenue, St Clair Shores, MI 48080 (E-mail: yveenap@yahoo.com). and appetite disturbances, decreased concentration, feelings of inadequacy as a parent, and despondent mood. Prevalence varies depending on how soon after delivery a woman is screened and which screening method is used, and may range from $10 \%$ to $20 \%$ of women. ${ }^{1-3}$ Several screening instruments have been developed, but the extensively validated, 10-question Edinburgh Postpartum Depression Scale (EPDS) ${ }^{4}$ is often the tool used to raise clinical suspicion of PPD. Nevertheless, it is estimated that fewer than half of PPD cases are recognized. ${ }^{5}$

Risk factors for PPD are numerous. In a metaanalysis, Beck ${ }^{6}$ identified 13 significant risk factors 
for postpartum depression. Ten factors had moderate effect size, including prenatal depression, selfesteem, childcare stress, prenatal anxiety, life stress, social support, marital relationship, history of depression, infant temperament, and maternity blues. Three factors had small effect size, including marital status, socioeconomic status (SES), and unplanned pregnancy. Of studies examining the association between SES status and PPD, few have reported that lower SES was predictive of PPD. ${ }^{7}$ Women with a history of depression are at increased risk for PPD. ${ }^{8}$

PPD has significant consequences for the woman and her family. At least one-fourth of women with a history of PPD will have a recurrence after another childbirth. ${ }^{9}$ Mothers with depressed moods may have difficulty responding to their infants, which can interfere with attachment. ${ }^{10}$ Other studies have found that children of depressed women exhibit behavioral disturbances ${ }^{11}$ and impaired cognitive development. ${ }^{12}$ Therefore, early identification and intervention of PPD is crucial.

Seasonally recurrent mood disorder, commonly known as seasonal affective disorder (SAD), has been documented for the last 20 years, with a lifetime prevalence of $0 \%$ to $9.7 \%$ in the general population. ${ }^{13}$ Symptoms include a depressed mood and a characteristic cluster of physical symptoms such as decreased activity, change in appetite, weight gain, decreased libido, and increased sleep duration. Episodes of depression tend to occur during specific times of the year, usually in winter. Women are approximately 4 times as likely as men to experience a seasonal variation in mood. ${ }^{14}$ This may be influenced by reproductive hormones; SAD increases after puberty and declines after menopause.

Given the number of research studies about $\mathrm{PPD}$ and $\mathrm{SAD}$, it is surprising that there has been little research conducted examining seasonal variation in PPD. Hiltunen et $\mathrm{al}^{15}$ conducted a study in Finland. The EPDS was used to measure level of depression in women immediately after delivery and 4 weeks postpartum. There was a greater incidence of mild PPD in the autumn (September through November) immediately after delivery, and less depression in the spring (March through May), measured 4 months postpartum. Dividing the data into daylight categories, women were more depressed during periods of limited sunlight.
In addition, mothers who were depressed immediately after delivery were more likely to be depressed later. This study supports the notion of seasonal variation in PPD, but it is unclear whether the results could be replicated in a population outside of Finland, a country that experiences extreme seasonal variation of natural light and dark.

This study attempted to define the significance of seasonal variation as a potential risk factor for PPD. We designed this pilot study to address the null hypothesis: seasonal variation in PPD (when measured using the EPDS between 5 to 8 weeks after delivery) is not a risk factor for PPD.

\section{Methods}

This cross-sectional study was conducted at 2 family medicine clinics and one obstetrics/gynecology clinic in the Detroit, Michigan metropolitan area, which is in the midwest United States, a region that observes daylight savings time. Data were collected from July 2004 through June 2006. All 3 sites included patients from a range of SESs. The 4 seasons were defined as Winter (December-February); Spring (March-May); Summer (June-August); and Fall (September-November). The St. John Hospital Institutional Review Board approved the study protocol.

A trained research assistant identified women who qualified for participation in the study. Potential patients included women who had given birth during the past 5 to 8 weeks, were able to complete the questionnaire, signed a written consent form, and were patients at one of the clinic sites. Women were excluded from participating in the study if they had given birth outside the range of the postpartum period (5-8 weeks), were unable to understand or complete the questionnaire, or had a stillborn child or infant death with their most recent pregnancy.

Physicians were educated about the study objectives, screening, and educational and referral materials before patient recruitment. The research assistant approached potential participants while they were waiting to be seen by their physician for the postpartum visit. After giving consent, each participant was asked to complete a demographic survey and the English version of the EPDS. The demographic survey included questions about the mother's age, income, education level, infant's birth weight, the extent of their social support, history of 
depression, and antidepressant medications. The EPDS is a 10-item, well-validated instrument highly effective in detecting PPD. ${ }^{1}$ It is the most sensitive and effective screening tool for PPD. ${ }^{4}$ EPDS items are scored from 0 to 2. An elevated EPDS score is not equivalent to the diagnostic criteria for PPD, but women with EPDS scores $>12$ are considered most likely to be diagnosed with PPD. ${ }^{4}$

When data collection was completed the women were given an educational brochure describing mood variation after delivery, a list of support services in the community, and inexpensive toys for their infant. When the patient scored $>12$ on the EPDS, or answered the suicidal ideation question positively, the research assistant alerted the patient's physician. The physician then discussed the screening results with the patient, determined if the clinical diagnosis of PPD was confirmed, and recommended treatment options.

Data were coded with a unique identifier for each mother and analyzed using SPSS software for Windows (SPSS, Inc., Chicago, IL). The $\chi^{2}$ test was used for evaluating associations between categorical variables (such as marital status and ethnicity) and unpaired $t$ test for continuous variables (mother's age and infant birth weight), comparing women who scored $>12$ (depressed) versus mothers who scored $<13$ (not depressed). Twins were excluded from the birth weight analysis $(n=6)$. The occurrence of PPD was analyzed by birth season and also by birth month. Logistic regression was used to find significant predictors of an EPDS score $>12$ (depression). Candidate variables evaluated by logistic regression included season of the year, extent of social support, site of prenatal care, social situation, marital status, race, prior diagnosis of depression, whether the mother was currently taking medication for depression, mother's age, infant birth weight, parity, and gravidity.

\section{Results}

Five hundred fifty-six potential participants were approached and 548 were recruited (99\% response rate). Ten were excluded as outside the time frame from delivery, and 530 completed the EPDS. The mean $( \pm \mathrm{SE})$ age of the sample was $24.9 \pm 0.2$ years; $71 \%$ were African Americans; $74 \%$ were single mothers; and $39 \%$ had at least some college education (Table 1). Of the sample, $17.8 \%$ scored
Table 1. Demographic Characteristics of the Study Postpartum Sample $(\mathbf{n}=\mathbf{5 3 0})$

\begin{tabular}{|c|c|}
\hline Variable & Value \\
\hline Age (mean \pm SE [range $]$ ) & $24.9 \pm 0.2(14-43)$ \\
\hline Gravidity (mean $\pm \mathrm{SE}$ [range]) & $2.1 \pm 0.1(0-10)$ \\
\hline Parity (mean $\pm \mathrm{SE}$ [range]) & $1.6 \pm 0.1(0-8)$ \\
\hline \multicolumn{2}{|l|}{ Marital Status $(\mathrm{n}=528)(\%)$} \\
\hline Single & 74 \\
\hline Married & 26 \\
\hline \multicolumn{2}{|l|}{ Ethnicity (\%) } \\
\hline African American & 71 \\
\hline White & 25 \\
\hline Other & 4 \\
\hline \multicolumn{2}{|l|}{ Location of obstetric care (\%) } \\
\hline $\mathrm{Ob} / g y n$ clinic & 81 \\
\hline Family medicine clinics & 11 \\
\hline Other sites & 5 \\
\hline No prenatal care & 3 \\
\hline \multicolumn{2}{|l|}{ Education (n = 332) (\%) } \\
\hline Some high school & 23 \\
\hline High school grad & 38 \\
\hline Some college & 30 \\
\hline College graduate school & 9 \\
\hline \multicolumn{2}{|l|}{ Annual ncome $(\mathrm{n}=459)(\%)$} \\
\hline$<\$ 10,000$ & 33 \\
\hline$\$ 10,000-24,000$ & 34 \\
\hline$\$ 25,000-49,000$ & 21 \\
\hline$>\$ 50,000$ & 12 \\
\hline
\end{tabular}

$>12$ on the EPDS. Season was not associated with the occurrence of PPD. Of patients who scored $>12$ on the EPDS, $18.1 \%$ had babies born during the winter, $19.2 \%$ had babies born during the spring, $13.4 \%$ had babies born during the summer, and $21.5 \%$ had babies born during fall $\left(\chi^{2} ; P=\right.$ .342 ; see Figure 1). Depression was lower in women giving birth during the summer (13.4\%) compared with all other seasons $(19.6 \% ; P=.097)$.

We found significant associations for a number of demographic variables that could affect PPD. Mothers with very good or excellent support at home had lower rates of PPD (12.6\%) than did mothers with adequate support $(44.0 \%)$ or very little or no support $(30.8 \% ; P<.0005)$. Mothers with high scores on the EPDS were of higher gravidity $(2.8 \pm 0.2$ vs $1.9 \pm 0.1 ; P=.001)$ and parity $(2.3 \pm 0.2$ vs $1.5 \pm 0.7 ; P<.0005$; see Figure $2)$. As expected, a greater proportion of women with a history of depression ( $\mathrm{n}=77 ; 42.9 \%$ ) versus those without a history of depression $(n=442$; $12.9 \%$ ), or those women currently taking antide- 


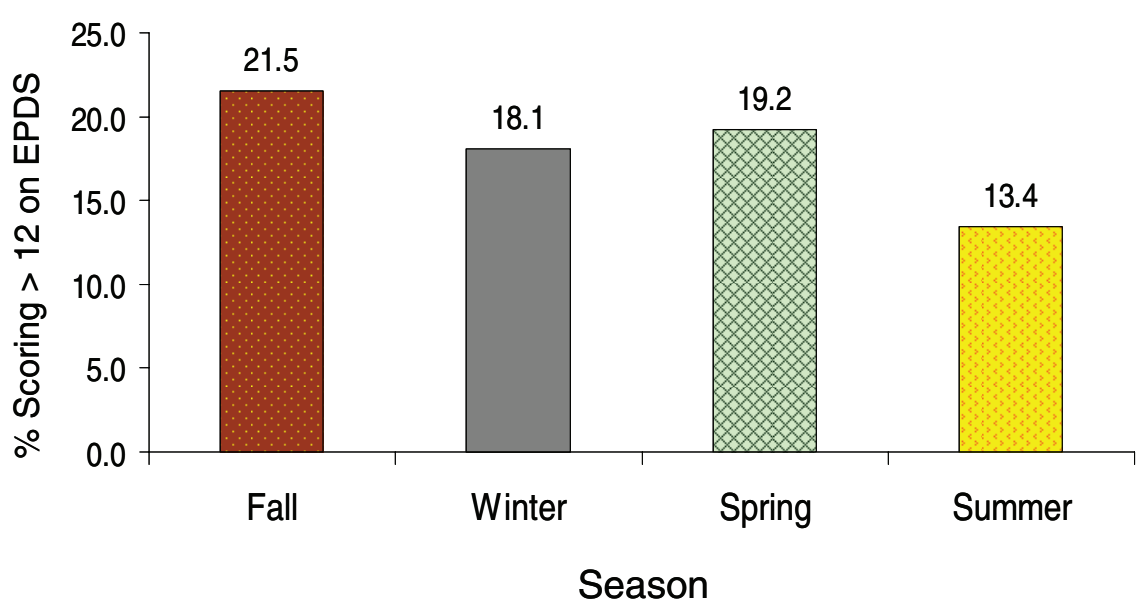

Figure 1. Incidence of postpartum depression, indicated by a score $>12$ on the Edinburgh Postpartum Depression Scale, during different seasons of the year $\left(\chi^{2} ; P=.342\right)$.

pressants $(\mathrm{n}=24 ; 58.3 \%)$ versus those women not currently taking antidepressants $(\mathrm{n}=503 ; 15.9 \%)$, had PPD $(P<.0005$; Figure 3$)$. When women with a prior or current indication of depression were excluded, the univariate results did not change. Mothers who scored $16.8 \pm 0.3$ (depressed) versus $4.8 \pm 0.2$ (not depressed) on the EPDS also had smaller babies $(3081.5 \pm 84.6 \mathrm{~g}$ vs $3285.6 \pm 34.8 \mathrm{~g}$, respectively; $P=.016$ ).

Logistic regression analysis, with education and income excluded (excess missing data), on the 452 women with complete datasets found 4 significant predictors of an EPDS score $>12$ (Table 2). The values, odds ratios, and $95 \%$ CIs for history of depression, parity, social support, and taking medication for depression are provided in the table.

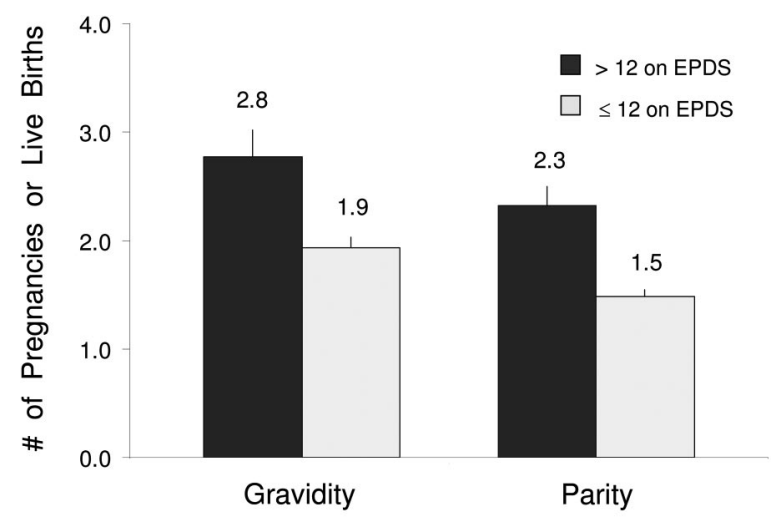

Reproductive History

Figure 2. Women of higher gravidity and parity had higher scores (dark bars) on the Edinburgh Postpartum Depression Scale ( $t$ test; $P<.0005)$.

\section{Discussion}

Our study yielded results that were consistent with the findings of previous studies. The rate of PPD in our population was $17.8 \%$, on the high side of the normal range for a typical postpartum patient population. Hobfoll et $\mathrm{al}^{16}$ also found higher rates of PPD among inner-city women. We did not find any seasonal variation when we compared 3-month periods (Summer, Spring, Fall, and Winter) or when we compared Summer with the rest of the year, although there was a lower incidence during Summer (13.4\%). This lower incidence of PPD during Summer is consistent with the expectation that women would be less likely to experience depression during months of abundant light. In our patients, the differences in levels of depression be-

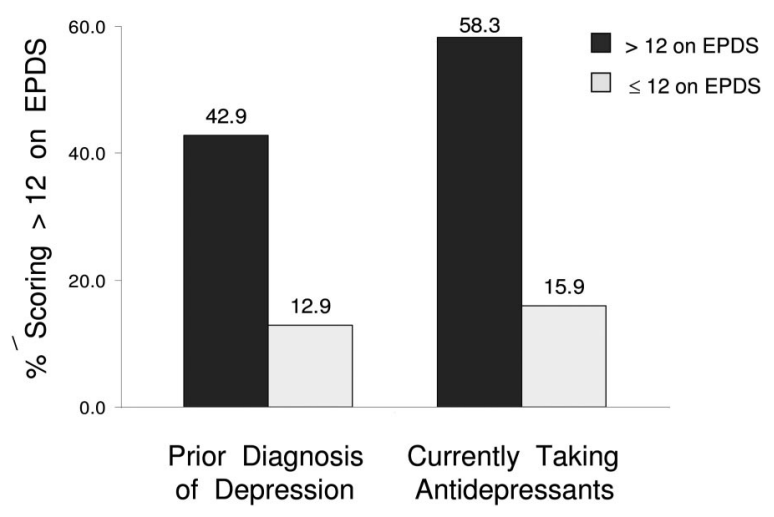

Figure 3. New mothers with a history of depression or who were taking antidepressive medication when they filled in the Edinburgh Postpartum Depression Scale were more likely to score $>\mathbf{1 2}$ on the Edinburgh Postpartum Depression Scale $\left(\chi^{2} ; P<.0005\right)$. 
Table 2. Predictors of Postpartum Depression from the Logistic Regression

\begin{tabular}{llll}
\hline Variable & Percent & Odds Ratio & $95 \%$ CI \\
\hline Depression & & & $2.016-7.949$ \\
$\quad$ Previous diagnosis of depression & 42.9 & 4.003 & 1.0 \\
$\quad$ No history & 12.9 & & $1.207-10.817$ \\
Antidepressives & 58.3 & 3.613 & 1.0 \\
$\quad$ Taking medication for depression & 15.9 & & $0.207-7.895$ \\
$\quad$ Not taking medication for & & 1.278 & $2.080-7.325$ \\
depression & 30.8 & 3.904 & 1.0 \\
Social support & 44.0 & 1.431 & $1.204-1.701$ \\
$\quad$ Not very good or little/none & 12.6 & & \\
$\quad$ Adequate & & & \\
$\quad$ Excellent or very good & $2.3 \pm 0.2$ & \\
Parity (mean \pm SE) & $1.5 \pm 0.1$ & & \\
$\quad$ Women with EPDS scores $\geq 13$ & & & \\
$\quad$ Women with EPDS scores $\leq 12$ & & & \\
\hline
\end{tabular}

EPDS, Edinburgh Postpartum Depression Scale.

tween seasons were less than those found by Hiltunen et al. ${ }^{15}$ Because we did find that social support, parity, history of depression, and currently taking medication for depression predicted the occurrence of PPD, it is clear that any seasonal effect was not as strong an influence in our population as these other factors. Thus, physicians should screen patients carefully for PPD during all seasons of the year.

Mothers who admit to having little or no support at home in caring for their babies should be closely monitored for signs of depression. This result is consistent with other studies. ${ }^{2}$ Women with a previous diagnosis of depression or who are currently taking antidepressants are at significant risk of developing $\mathrm{PPD}^{2}$ and should be carefully evaluated after delivery. They may require close monitoring of antidepressants so that dosing could be titrated up when needed and/or additional counseling could be recommended. In addition, mothers could be educated during pregnancy about risk factors so that they are aware of the possibility of developing PPD. Physicians could suggest that, if possible, at-risk mothers work to improve their support system.

Women with a history of depression and those taking antidepressants are of particular concern. Some women discontinue medications during pregnancy or breastfeeding but should discuss the risks and benefits with their physicians. In addition, depressed postpartum women may need additional mental health services, including psychiatric evalu- ation, psychotherapy, support groups, or hospitalization. ${ }^{9}$ Women being treated for depression during the prenatal period should be encouraged to discuss the management of their depression with their physician during and after pregnancy. The mood changes that occur during this time should be taken into account and treatment should be modified as needed. One investigation found morning light therapy to be advantageous for depressed pregnant women. ${ }^{17}$ Women with a history of depression who have discontinued treatment should be made aware of their increased risk of PPD. The significant associations found with social stressors, larger family size, history of depression, and currently taking antidepressants suggests that new mothers with these characteristics should definitely be questioned about PPD symptoms.

\section{Limitations}

Our study had several limitations. First of all, the small sample size lowered the power of our study. The preponderance of African American women in our study did not reflect the US population. We would need a more diverse sample of whites and Hispanics of varying SES to appropriately represent the present US population. Our inability to detect a difference in PPD by season is probably because of one of 2 factors: there may be no seasonal variability in PPD or we had too few patients to detect variability. Our prestudy power calculation based on the seasonal variation found by Hiltunen et $\mathrm{al}^{15}$ suggested that we would need 245 
women per group to show a significant difference; we had enrolled only 149 patients during the summer and 144 during the winter. Based on this data, we would need 3 times the size of the present population to determine whether the observed difference was significant.

\section{Conclusion}

The slight seasonal variation in PPD in our study was not statistically significant, but the study was underpowered for the difference in PPD rate we detected. Stronger associations with increased PPD rate were observed for social stressors, multigravidity, history of depression, and currently taking antidepressants. Additional sites are needed to provide greater variation in the participants from geographic locations, marital status, SES, and racial backgrounds to diversify and increase the sample size and to provide adequate statistical power to test projected seasonal variations.

\section{Recommendations}

The high odds ratios found for social support, gravidity, history of depression, and currently taking antidepressants suggest that new mothers with these characteristics should definitely be questioned about PPD symptoms.

It is recommended that clinicians be on alert to detect this problem in mothers:

- during all seasons;

- who have more children;

- who lack a good support system;

- with a history of depression; and

- who are currently taking antidepressants.

The authors extend their appreciation to Karen Hagglund, MS, for her assistance in the data analysis, and to Paul Paonessa, MD; George Maristela, MD; and Patricia Mackin for their help with data collection.

\section{References}

1. Cox JL, Chapmand G, Murray D, Jones P. Validation of the Edinburgh Postnatal Depression Scale (EPDS) in non-postnatal women. J Affect Disord 1996;39:185-189.
2. O'Hara MW, Swain AM. Rates and risks of postpartum depression: a meta-analysis. Int Rev Psychiatry 1996;8:37-54.

3. Steiner M. Perinatal mood disorders: position paper. Psychopharmacol Bull 1998;34:301-6.

4. Cox JL, Holden JM, Sagovsky R. Detection of postnatal depression. Development of the 10-item Edinburgh Postnatal Depression Scale. Br J Psychiatry 1987;150:782-6.

5. Gjerdingen DK, Yawn BP. Postpartum depression screening: importance, methods, barriers, and recommendations for practice. J Am Board Fam Med 2007;20:280-8.

6. Beck CT. Predictors of postpartum depression: an update. Nurs Res 2001;50:275-85.

7. O'Hara MW, Rehm LP, Campbell SB. Postpartum depression: a role for social network and life stress variables. J Nerv Ment Dis 1983;171:336-41.

8. Epperson CN. Postpartum major depression: detection and treatment. Am Fam Physician 1999;59: 2247-59.

9. Wisner KL, Parry BL, Piontek CM. Postpartum depression. N Engl J Med 2002;347:194-9.

10. Beck CT. The effects of postpartum depression on maternal-infant interactions: a meta-analysis. Nurs Res 1995;44:298-304.

11. Weinberg MK, Troick EZ. Maternal depression and infant maladjustment: a failure of mutual regulation. In: Nospitz JD, ed. Handbook of child and adolescent psychiatry. New York, NY: John Wiley \& Sons, Inc.; 1997:243-57.

12. Cogill SR, Caplan HL, Alexandra H, Robson KM, Kumar R. Impact of maternal postnatal depression on cognitive development of young children. BMJ 1986;292:1165-7.

13. Lurie SJ, Gawinski B, Pierce D. Seasonal affective disorder. Am Fam Physician 2006;74:1521-4.

14. Rosenthal NE. Diagnosis and treatment of Seasonal Affective Disorder. J Am Med Assn 1993;270:271720.

15. Hiltunen P, Jokelainen J, Ebeling H, Szajnberg N, Moilanen I. Seasonal variation in postnatal depression. J Affect Disorders 2004;78:111-8.

16. Hobfoll SE, Ritten C, Lavin J, Hulsizer MR, Cameron RP. Depression prevalence and incidence among inner-city pregnant and postpartum women. J Consult Clin Psychol 1995;63:445-53.

17. Oren DA, Wisnes KL, Spinelli M, et al. An open trail of morning light therapy for treatment on antepartum depression. Am J Psychiatry 2002;159: $666-9$. 